\title{
Photonics meets excitonics: natural and artificial molecular aggregates
}

\section{Citation}

Saikin, Semion K., Alexander Eisfeld, Stéphanie Valleau, and Alán Aspuru-Guzik. 2013.

"Photonics Meets Excitonics: Natural and Artificial Molecular Aggregates." Nanophotonics 2, no. 1: 21-38.

\section{Published Version}

doi:10.1515/nanoph-2012-0025

\section{Permanent link}

http://nrs.harvard.edu/urn-3:HUL.InstRepos:12362622

\section{Terms of Use}

This article was downloaded from Harvard University's DASH repository, and is made available under the terms and conditions applicable to Other Posted Material, as set forth at http:// nrs.harvard.edu/urn-3:HUL.InstRepos:dash.current.terms-of-use\#LAA

\section{Share Your Story}

The Harvard community has made this article openly available.

Please share how this access benefits you. Submit a story.

\section{Accessibility}




\section{Review article}

\section{Semion K. Saikin*, Alexander Eisfeld*, Stéphanie Valleau and Alán Aspuru-Guzik* Photonics meets excitonics: natural and artificial molecular aggregates}

\begin{abstract}
Organic molecules store the energy of absorbed light in the form of charge-neutral molecular excitations Frenkel excitons. Usually, in amorphous organic materials, excitons are viewed as quasiparticles, localized on single molecules, which diffuse randomly through the structure. However, the picture of incoherent hopping is not applicable to some classes of molecular aggregates - assemblies of molecules that have strong near-field interaction between electronic excitations in the individual subunits. Molecular aggregates can be found in nature, in photosynthetic complexes of plants and bacteria, and they can also be produced artificially in various forms including quasi-one dimensional chains, two-dimensional films, tubes, etc. In these structures light is absorbed collectively by many molecules and the following dynamics of molecular excitation possesses coherent properties. This energy transfer mechanism, mediated by the coherent exciton dynamics, resembles the propagation of electromagnetic waves through a structured medium on the nanometer scale. The absorbed energy can be transferred resonantly over distances of hundreds of nanometers before exciton relaxation occurs. Furthermore, the spatial and energetic landscape of molecular aggregates can enable the funneling of the exciton energy to a small number of molecules either within or outside the aggregate. In this review we establish a bridge between the fields of photonics and excitonics by describing the present understanding of exciton dynamics in molecular aggregates.
\end{abstract}

Keywords: exciton; exciton dynamics; molecular aggregates; J-aggregates; H-aggregates; light-harvesting complexes.

\footnotetext{
*Corresponding authors: Semion K. Saikin and Alán Aspuru-Guzik, Department of Chemistry and Chemical Biology, Harvard University, 12 Oxford Street, Cambridge, MA 02138, USA, e-mail: saykin@fas.harvard.edu; aspuru@chemistry.harvard.edu; and Alexander Eisfeld, Max-Planck-Institut für Physik komplexer Systeme, Nöthnitzer Str. 38, D-01187 Dresden, Germany, e-mail: eisfeld@mpipks-dresden.mpg.de Semion K. Saikin: Department of Physics, Kazan Federal University, 18 Kremlyovskaya Street, Kazan 420008, Russian Federation Stéphanie Valleau: Department of Chemistry and Chemical Biology, Harvard University, 12 Oxford Street, Cambridge, MA 02138, USA

Edited by Volker Sorger
}

\section{Introduction}

Advances in nanotechnology supported by our understanding of material properties on the microscopic level persistently drive the field of photonics to the nanometer scale. With the development of photonic crystals $[1,2]$ and semiconductor optical cavities $[3,4]$ the size of optical devices, usually limited by light diffraction in free space, can been scaled down to hundreds of nanometers just by exploiting the material's dielectric constant. Advances in the design of plasmonic metamaterials [5-7] permitted this limit to be pushed even further down to the order of tens of nanometers. The natural question which arises is: what will be the next limit and how can it be approached?

In this review we consider one of possible pathways towards reaching the goal of optical devices on the nanometer scale: using molecular aggregates as photon processing elements on the true nanometer scale, see Figure 1. Molecular aggregates are assemblies of molecules held in place by non-covalent interactions. These single molecules mostly keep their nuclear and electronic structure. Examples are molecular crystals as well as nanoscale self-assembled structures, molecular films and light harvesting systems in photosynthesis. If the lowest allowed electronic transitions in the composing molecules are within the visible part of the optical spectrum and if the molecules have large absorption and fluorescence cross-sections, the interaction between molecular electronic transitions is strong enough to transfer the light absorbed from one molecule to the others, via a resonant de-excitation/excitation process.

This mechanism is closely related to the near-field energy transfer between classical dipoles (antennas) [8-13]. In this sense, the exciton dynamics in molecular aggregates resembles the propagation of light in a metamaterial where dye molecules play the role of functional elements. In contrast to excitons in inorganic semiconductors, where free charge carrier mobility determines the exciton transport, in aggregates, electrons remain localized on molecules while the excitation is transferred.

A canonical example of molecular aggregates that possess coherent exciton properties are J-aggregates. 

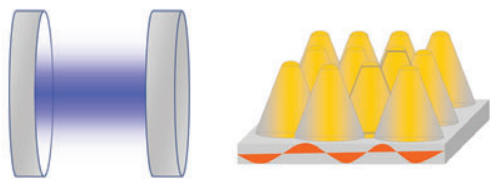

Photonics

Plasmonics

Excitonics

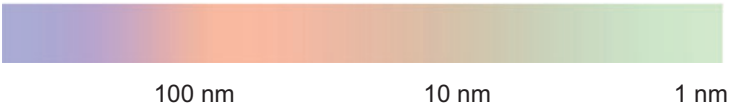

Figure 1 Schematic illustration of the length scales characterizing the different physical phenomena present the different fields discussed in this review.

These are aggregates of fluorescent molecules, discovered about 80 years ago independently by Scheibe $[14,15]$ and Jelley [16]. They observed that the formation of aggregates is accompanied by drastic changes in the optical properties. The broad absorption line corresponding to the molecular excitation is shifted to the red side of the spectrum and becomes much narrower. Later, these types of molecular aggregates were named J-aggregates after Jelley. J-aggregates represent only one class of molecular aggregates. For instance, there exist aggregates where the absorption line is blue-shifted, so called $\mathrm{H}$-aggregates. Some aggregates exhibit several J-bands [17] and some have both J-and H-bands [18]. In aggregates that consist of non-equivalent monomers even more complicated absorption structures can be found [19].

Molecular aggregates also appear as functional units in nature. For instance, they form the absorbing and energy transferring parts of light-harvesting complexes in plants and some types of bacteria and algae [19]. In these complexes the exciton absorbed by the antenna aggregate has to be funneled to the reaction center - the part where the exciton energy is used to create free charges to be employed in a chemical reaction. The efficiency and robustness of light absorption and exciton transfer in light harvesting complexes may be crucial for the survival of photosynthetic organisms under evolutionary pressure.

Shortly after the discovery of the self-assembled organic dye aggregates the close connection of these artificial structures to the natural light harvesting systems was recognized $[20,21]$ and the exciton model of Frenkel $[9,22]$ has been used to explain the observed changes in optical properties and the transfer of energy along the aggregate [21].

In Frenkel's exciton theory, which is based on the classical resonance interaction theory of Holtsmark [8], the electronic excitation in the aggregate is not confined to a single monomer, but it is coherently delocalized over many monomers in the form of "excitation waves".
From superpositions of these excitation waves "excitation packets" can be formed, which describe the coherent motion of (localized) excitations [22]. Already in these early works it was established that coupling to internal and external vibrational modes, imperfections of the aggregates and disorder induced by the environment strongly influences the "coherence size" of the exciton waves and modifies the optical and transport properties.

When the interaction with the environment (internal vibrations, solvent, etc.) is much stronger than the resonant excitation transfer interaction, the excitation becomes more or less localized on one monomer and the transfer is no longer described by coherent exciton motion but it becomes an incoherent hopping process. Förster derived an elegant formula for the rate constants for transport of excitation from one monomer to the other [23, 24]. This rate is proportional to the overlap of the donor emission spectrum and the absorption spectrum of the acceptor molecule and depends on the inverse sixth power of the distance between donor and acceptor. Typically, in molecular aggregates, one is in a regime in-between these two extreme cases, i.e., the transport is neither fully coherent nor incoherent. This complicates the theoretical modeling and the interpretation of experiments.

The remarkable optical and transport properties of molecular aggregates have led to a variety of applications. Right from the beginning, molecular aggregates were employed as wavelength selective sensitizers in photography $[25,26]$. Recent applications include the measurement of membrane potentials [27] or the design of colorants [28]. Some molecular aggregates form self-assembled supramolecular flexible fluorescent fibers [29] which may have applications in thin-film optical and optoelectronic devices, for example by employing optical bistability of J-aggregates [30]. Molecular aggregates can be also utilized for sensing applications. For instance, it has been demonstrated that a large absorption cross section combined with fast exciton diffusion may be used to enhance fluorescence from a small number of dye molecules adsorbed or embedded in an aggregate film [31, 32]. Due to fast exciton diffusion within the aggregate the excitons explore the aggregate. Once they find a molecule with an electronic transition close to the J-band the exciton can be transferred inelastically to the adsorbed molecule. Moreover, dye aggregates might play an important role in the development of efficient, low-cost artificial light harvesting units (like organic solar cells) [33].

This review gives a brief, yet by no means complete, overview of our experimental and theoretical understanding of excitons in molecular aggregates. Most of the aspects in this work are covered at the advanced introductory level, and for a deeper understanding we refer to more 
detailed studies such as [19, 34-38]. The review is structured as follows: In Section 2, we describe basic excitonic properties of molecular aggregates starting with single molecules and molecular dimers and then introducing several examples of artificial and natural molecular aggregates. In Section 3, we overview the main experimental techniques that allow for probing the structural properties of molecular aggregates and excitation dynamics. Section 4 introduces the main theoretical approaches utilized for modeling of excitons in aggregates. Section 5 shows how molecular aggregates can be combined with photonic and plasmonic structures. Finally, we conclude the review in Section 6.

\section{Basic properties of molecular aggregates}

\subsection{Properties of monomers}

Molecules in the aggregates largely retain their electronic and nuclear structure. Thus, it is natural to start our discussion with individual molecules interacting with light. The interaction of a single molecule with light is schematically illustrated in Figure 2. If the molecule has an optically allowed electronic transitions within the photon spectrum it can be excited by absorbing light at the corresponding frequency. The transition time between the ground and excited state of the molecule is of the order of tens of attoseconds - the time that a single photon interacts with a molecule. During this short time interval the positions of the nuclei in the molecule are not changed. Initially the molecule is in its ground state geometry, i.e., in a minimum of the electronic ground state potential $|g\rangle$ indicated by the blue wave-packet in Figure 2. The equilibrium positions of atoms for the excited states, usually, are different from those of the ground state. Therefore, after the transition to the state $|e\rangle$ the molecule is in a transient non-equilibrium state where molecular vibrational modes are also excited (green wave-packet in Figure 2). Then, due to the interaction of the molecule with its environment the molecule relaxes towards the energetic minimum of the excited state, i.e., the excited vibrational modes are relaxed (wavy arrow and orange wavepacket in Figure 2). This relaxation of the molecular geometry can also be accompanied by a rearrangement of the environmental molecules to a configuration with lower total energy. At ambient conditions this process occurs over a timescale of hundreds of femtoseconds to several picoseconds.

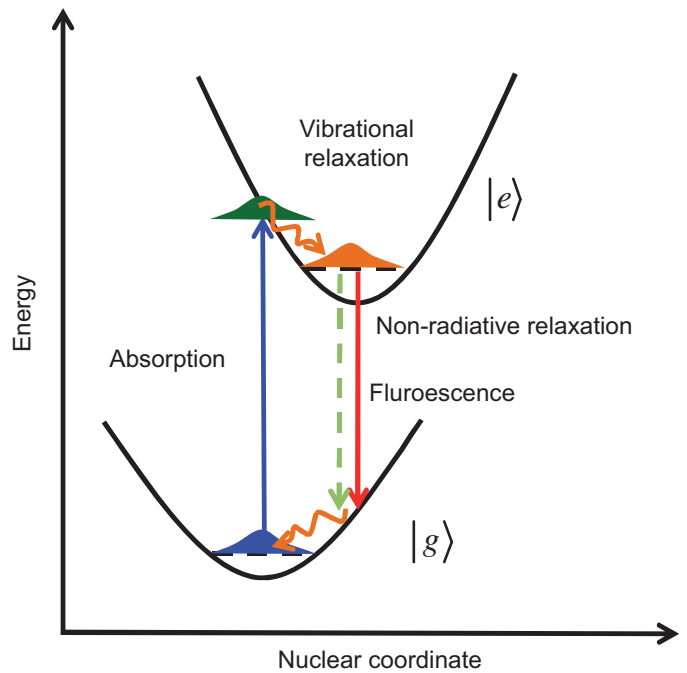

Figure 2 Excitation/de-excitation processes in a single molecule illustrated on a two-level molecular energy diagram. The parabolic surfaces correspond to the ground state $|g\rangle$ and the first excited $|e\rangle$ electronic states of the molecule. The horizontal axis indicates the displacements of nuclei from their equilibrium positions. The molecule, initially in the ground electronic and vibrational states, is excited by absorbing light - blue arrow. During the absorption process the positions of the nuclei are not changed. The light absorption also induces molecular vibrations. Due to the interaction with the environment the molecular vibrations are equilibrated and the molecule relaxes to the bottom of the excited electronic state - orange wavy arrow. Finally, the molecule relaxes down to the ground electronic state by emitting a photon - red arrow (fluorescence), or without photon emission - green dashed arrow (non-radiative process). In the ground state the induced vibrations are also equilibrated due to interaction with the environment.

Finally, on timescales of tens of picoseconds to nanoseconds the molecule relaxes to its electronic ground state either emitting a photon (fluorescence) or transferring energy to other degrees of freedom, for instance vibrations (non-radiative relaxation [39]). Usually, the absorption and emission spectra exhibit a progression of peaks stemming from the coupling to vibrational modes with high energy $(\sim 150 \mathrm{meV})$. These peaks are broadened by the same order of magnitude, due to coupling to a multitude of low energy modes of the molecule and the surroundings. Emission takes place from the thermally relaxed excited state, which is typically located in the low energy wing of the absorption spectrum. The relaxation energy is related to the energy difference between the maxima of the absorption and emission spectra, the so-called Stokes shift.

The absorption efficiency of a particular electronic transition can often be characterized by the corresponding transition dipole - a matrix element of a dipole operator between the ground and the excited molecular states. 


$$
\vec{d}=\langle e|q \cdot \vec{r}| g\rangle .
$$

Here and in the following we use an arrow symbol to indicate three-dimensional vectors in real space. The dipole operator is characterized by its charge $q$ and the position operator $\vec{r}$. The transition dipole is not a measurable value and is defined up to a complex phase factor (if magnetic interactions can be neglected, which is often the case, then the wavefunction can be chosen real and $\vec{d}$ becomes a real vector). The absorption and emission strengths of the respective transition are proportional to $|\vec{d}|^{2}$. In the absorption spectra the transition frequency is determined by the energy difference $\varepsilon$ between the ground and excited state.

Structures and optical spectra of representative molecules are shown in Figures 3 and 4. In Figure 3 we show a TDBC molecule, which can be used as an illustrative example for a broader class of cyanine dyes. Beside the monomeric absorption and fluorescence spectra (in solution), spectra of aggregates formed on a glass substrate are also shown. The lowest electronic transition in TDBC is characterized by a large transition dipole of the order of $|\vec{d}| \approx 10$ Debye (Debye units are commonly used for
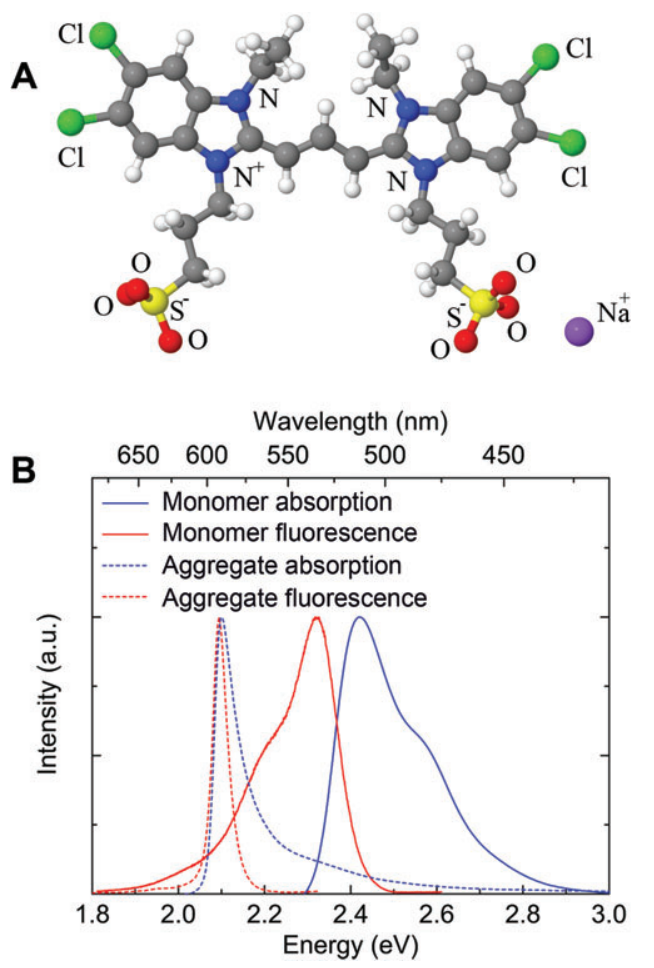

Figure 3 TDBC fluorescent dye that forms 1D and 2D molecular aggregates. (A) Molecular structure, gray and white spheres represent carbons and hydrogens respectively. (B) Normalized absorption and fluorescence spectra of TDBC molecules taken in a solution (solid lines) and in a 2D aggregated form (dashed lines). molecular dipoles, 1 Debye $\approx 0.208 \mathrm{e} \cdot \AA$ where $e$ denotes the electron charge), which is aligned with the backbone of the molecule. The higher excited states in TDBC are well separated from the lowest one, which indicates that, for low intensity optical absorption and exciton dynamics it is sufficient to take only two electronic states into account. The absorption spectrum of the molecule in a solution shows a broad line in the green part of the spectrum with a partially resolved vibronic structure. Note also that the emission from a monomer is roughly a mirror-image of the absorption, where the maximum is shifted to lower energies. This is the Stokes shift, which is caused by the relaxation/reorganization after an electronic transition. The mirror image of absorption and fluorescence indicates that the vibrational frequencies and their coupling to electronic transitions are very similar in the electronic ground and excited state. Upon aggregation the absorption and fluorescence lines become much narrower with no structure and are shifted to the orange-red color. The Stokes shift in the aggregated form is also much smaller, which indicates a reduced coupling to the environment. Aggregates composed of TDBC have, for example, been produced in solution [17] and on surfaces [40]. By changing the side groups different geometrical arrangements can be achieved [17].

Another representative example of molecules forming aggregates is the bacteriochlorophyll (Bchl) - a pigment molecule, which is a functional element of photosynthetic systems in phototrophic bacteria, see Figure 4(A). The structure of Bchl is similar to that of chlorophyll the photosynthetic pigment in plants. Both are derivatives of porphyrin, compelexed with $\mathrm{Mg}^{2+}$. The lowest electronic transition, which is involved in the energy transfer, is the so called $Q_{y}$-band. The corresponding transition dipole lies in the plane of the porphyrin ring and is about $\vec{d} \mid \approx 5$ Debye. The transition dipole corresponding to the next electronic state is denoted as $Q_{x}$. It is perpendicular to the $Q_{y}$ dipole, and the associated transition strength is much smaller. The Soret band which lies in the ultraviolet has stronger absorption but is usually not involved in the energy transfer. As in the case of TDBC the emission occurs from the lowest excited state and the emission spectrum is roughly a mirror image of the absorption band corresponding to this state.

\subsection{The aggregate}

Organic dye molecules can self-aggregate in different types of structures. Sometimes the aggregation is driven by electrostatic forces pushing molecules to adsorb on a 

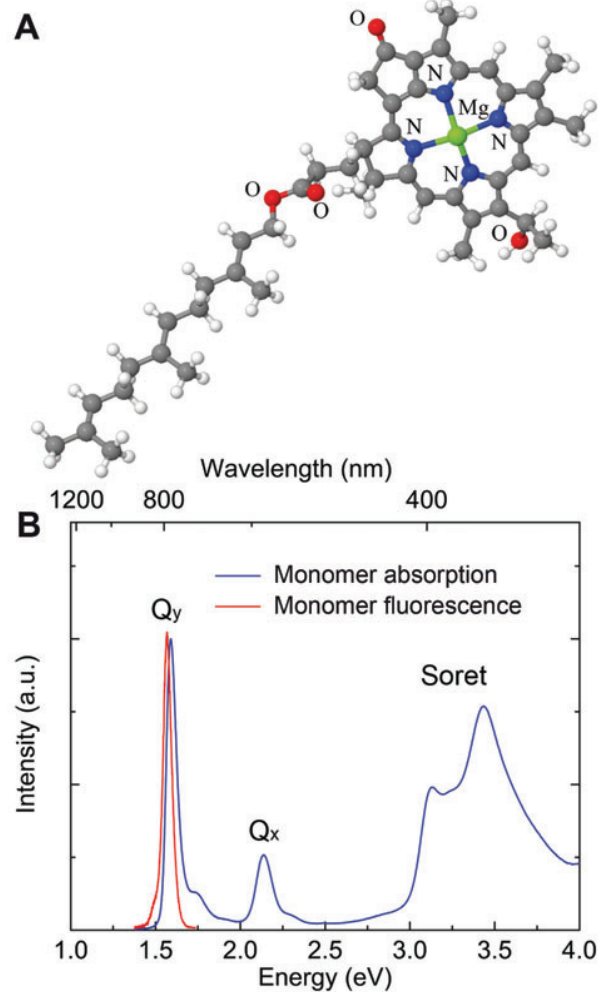

Figure 4 (A) Bacteriochlorophyll (Bchl) molecule - light absorbing pigment that is a basic structural element of light-harvesting complexes in phototrophic bacteria. Its structure is similar to chlorophyll - the photosynthetic pigment in plants.; (B) Absorption and fluorescent spectra of Bchl molecules.

surface; sometimes hydrophobic parts of the molecules repel the water and tend to collect together such as in synthetic tube aggregates [17].

A specific property of molecular aggregates with strong inter-monomer couplings is that the absorption and fluorescent spectra substantially differ from the spectra of the molecules which form the aggregates while the electronic structure of the molecules is not modified. The intermolecular distance in an aggregate is large enough that the electron tunneling between different molecules can be neglected. Therefore, only the Coulomb interaction between the electronic transitions in monomers is responsible for the spectral modifications and the intermolecular energy transfer. This interaction is similar to the non-radiative near-field coupling between plasmonic structures, which is mediated by virtual photons. It turns out that the major contribution of the Coulomb interaction in the spectra of aggregates is from the excitation transfer via resonant transitions. We will refer to this main interaction term between the monomers as Förster coupling. The Förster coupling, to a good approximation, can be described by restricting to dipole transitions only (see below Eq. 2). The interaction is visualized in Figure 5 for a situation in which initially monomer 1 is electronically excited and monomer 2 is in the electronic ground state. Then, in a resonant process molecule 1 is deexcited and simultaneously the second monomer is excited. While this time-ordered discrete picture is easy for visualization in reality the exciton energy is transferred coherently. For large distances between the monomers the interaction between monomers 1 and 2 can be approximated by the dipole-dipole term

$$
V_{12}=\frac{1}{R_{12}^{3}}\left(\vec{d}_{1} \cdot \vec{d}_{2}-3\left(\vec{d}_{1} \cdot \hat{R}_{12}\right)\left(\vec{d}_{2} \cdot \hat{R}_{12}\right)\right),
$$

where $R_{12}$ denotes the distance between the monomers, $\hat{R}_{12}$ is the corresponding direction vector, and the vectors $\vec{d}_{n}$ are the transition dipoles introduced in Eq. (1).

If the intermolecular distance is comparable with the size of the molecules, then the interaction between the two monomers can no longer be described adequately using the point dipole-dipole interaction of Eq. (2). Often, it is then sufficient to replace the point-dipoles in Eq. (2) by extended dipoles [41]. For even higher accuracy more elaborate schemes have been developed (see e.g., [42]).

Before discussing general molecular aggregates, let us describe some basic results for the case of two coupled identical monomers, ignoring nuclear degrees of freedom for the moment. The eigenstates of the uncoupled monomers can be taken as a basis. These states are $|g\rangle|g\rangle,|e\rangle|g\rangle$, $|g\rangle|e\rangle,|e\rangle|e\rangle$. While the states $|g\rangle|g\rangle$ and $|e\rangle|e\rangle$ are still suitable to describe the ground state and the doubly excited state of the dimer, respectively, the two degenerate single exciton states $|e\rangle|g\rangle$ and $|g\rangle|e\rangle$ are no longer eigenstates of the coupled system, because of the resonant transfer interaction. The corresponding Hamiltonian is written as $H=$ $\varepsilon_{1}|1\rangle\left\langle 1\left|+\varepsilon_{2}\right| 2\right\rangle\langle 2|+V_{12}(|1\rangle\langle 2|+| 2\rangle\langle 1|)$ (we choose the energy of the monomer ground state as zero), where we have introduced

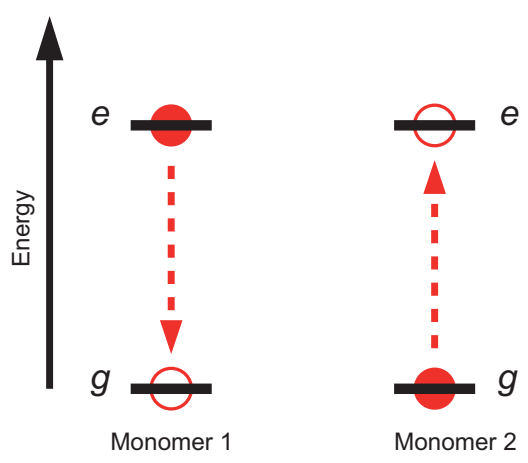

Figure 5 Illustration of the excitation transfer due to resonant near-field interaction between the state $|e\rangle|g\rangle$ and $|g\rangle|e\rangle$. 
the shorthand notation $|1\rangle \equiv|e\rangle|g\rangle$ and $|2\rangle \equiv|g\rangle|e\rangle$ or equivalently in a matrix representation

$$
H=\left(\begin{array}{cc}
\varepsilon_{1} & V_{12} \\
V_{12} & \varepsilon_{2}
\end{array}\right)
$$

For identical energies of excited states $\varepsilon_{1}=\varepsilon_{2} \equiv \varepsilon$ the eigenstates of the dimer are superpositions $| \pm\rangle=\frac{1}{\sqrt{2}}(|e\rangle|g\rangle \pm|e\rangle|g\rangle)$ where the electronic excitation is coherently delocalized over both monomers. The corresponding eigenenergies are $\varepsilon_{ \pm}=\varepsilon \pm V_{12}$. Note that the magnitude and sign of the interaction depends sensitively on the distance and orientation of the two monomers. Figure 6 illustrates how the relative orientations of molecular transition dipoles change the frequency of electronic excitations in a dimer. If the transition frequencies of the involved monomers are equal and the transition dipoles are parallel, only the transition between the ground and the $|+\rangle$ state is optically allowed. It is detuned by the energy $V_{12}$ from that of the non-interacting monomers. From Eq. (2) one can see that for case (a), where the transition dipoles are orthogonal to the distance vector between the monomers one has positive $V_{12} \sim|d|^{2} / R_{12}^{3}$. For the case (b) the interaction is negative with $V_{12} \sim-2|d|^{2} / R_{12}^{3}$. Therefore, the alinement of the molecules shown in Figure 6 (A) and (B) will be seen as a blue and red shift of the absorption line, respectively. As it has been noted before, the states with only one excited monomer such as $|g\rangle|e\rangle$ and
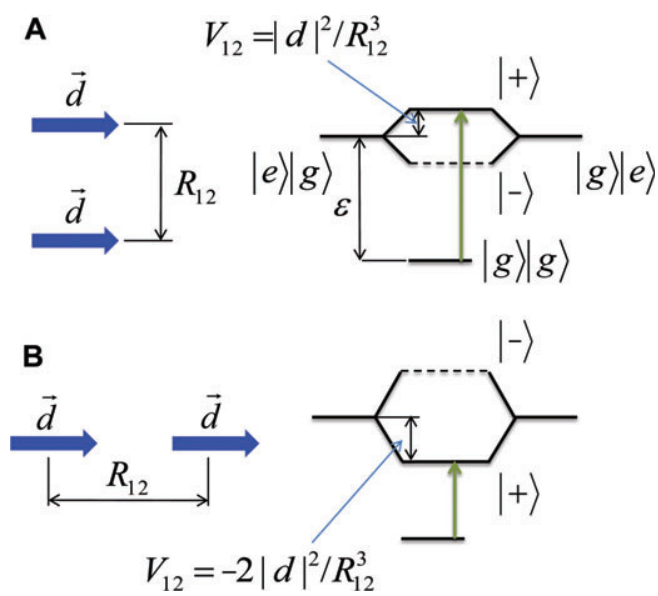

Figure 6 Interaction between electronic transitions dipoles (blue arrows) of two molecules. The directions of the arrows reflect the relative phase of the transition dipoles. For the considered molecular alignments only one of the optical transitions is allowed for the dimer (green arrows). (A) The optically allowed transition of the dimer is shifted to the blue part of the spectrum, which is similar to H-aggregation; (B) the optically allowed transition is shifted to the red, which correspond to J-aggregation. $|e\rangle|g\rangle$ are not eigenstates of the system anymore. Thus, if one of these state is populated initially, the energy will oscillate between them back and forth coherently.

Let us briefly mention that the dimer often appears as a first step of aggregation [43] and thus has been investigated intensively (see e.g., [44-49]) . This interest in the dimer is also due to the fact that many of the more realistic models for aggregates where the environment and the internal vibrations are included can only be solved efficiently for the dimer.

The theoretical description of the dimer can easily be extended to arbitrarily arranged monomers. As in the case of the dimer the total ground state is taken as a product of states where all monomers are in the ground state $\left|g_{\mathrm{el}}^{\mathrm{agg}}\right\rangle=|g\rangle_{1} \ldots|g\rangle_{N}$. We are interested in the properties of aggregates in the linear absorption regime. Therefore, it is sufficient to take into account only the states with at most one electronic excitation on the aggregate, such as

$$
|n\rangle \equiv|g\rangle_{1} \ldots|e\rangle_{n} \ldots|g\rangle_{N},
$$

in which monomer $n$ is electronically excited and all other monomers are in their electronic ground state. In this oneexciton manifold approximation the Hamiltonian can be written as

$$
H^{e}=\sum_{n=1}^{N} \varepsilon_{n}|n\rangle\left\langle n\left|+\sum_{n m} V_{n m}\right| n\right\rangle\langle m|
$$

where $V_{n m}$ is the Coulomb dipole-dipole interaction, see Eq. 2, which transfers excitation from monomer $n$ to $m$.

Examples of commonly discussed 1D and 2D planar structures are shown in Figure 7. While 1D models are well studied theoretically (see e.g., Refs. [50-58]) and are frequently used to characterize excitons in self-assembled molecular aggregates, it is not clear whether ideal 1D molecular chains are formed in experiments [34]. Usually, they are subsystems of higher dimensional structures such as films or crystals.

The 1D model of molecular aggregates is convenient because many results can be obtained analytically $[19,59]$. For a perfect very long chain with $N$ molecules the eigenstates are well described by "exciton waves"

$$
\left|\phi_{j}\right\rangle=\frac{1}{\sqrt{N}} \sum_{n=1}^{N} e^{i \frac{2 \pi}{N} j n}|n\rangle
$$

with the corresponding eigenenergies

$$
E_{j}=\varepsilon+2 V \cos \left(\frac{2 \pi j}{N}\right) .
$$

In the last equation, for the sake of simplicity, we have considered the interactions between nearest neighbors 
A

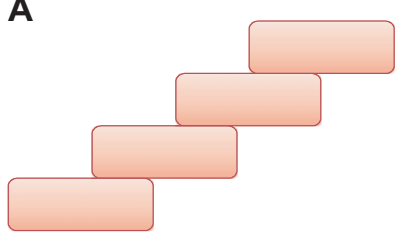

B

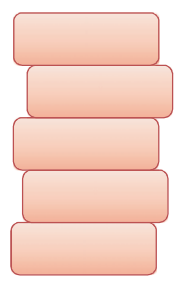

C

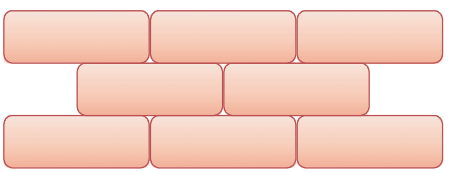

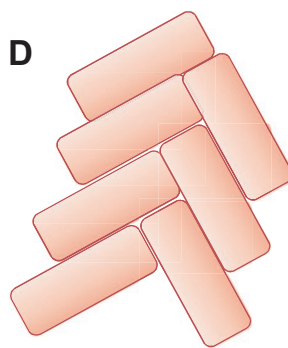

Figure 7 Illustrations of 1D and 2D planar molecular aggregates. Each block corresponds to a monomer forming the aggregate. (A) staircase and (B) ladder models for 1D packing; (C) brickstone and (D) herringbone 2D packing models.

only, which are denoted by $V$. A discussion of finite onedimensional aggregates can be found e.g., in Refs. [19, 59].

For the perfect linear chain with parallel monomers, the largest transition dipole correspond to the excitation of the state with a minimal number of nodes. In the case of J-aggregation, Figure 7A, this state is at the bottom of the exciton spectrum shifted by about several hundreds of $\mathrm{meV}$ from the monomer transition while for $\mathrm{H}$-aggregates, Figure $7 \mathrm{~B}$, this shift is to the blue part of the spectrum. In reality, to compare exciton transitions in aggregates with excitations in single molecules one also has to include Van der Waals interaction with off-resonant excitations $[19,47]$ and the vibronic structure of molecular excitation [19, 47, 60]. However, the simplified model presented above qualitatively describes the exciton states in aggregates.

2D molecular aggregates can be formed, for instance, as Langmuir-Blodgett films [61], deposited molecule-bymolecule on a surface [40,62], or by spin coating [63]. The structure of the aggregate is determined by the molecular properties such as their shape, charge, etc., and the assembly method. For instance, for cyanine dyes [64] (TDBC is one example of the class of molecules) some commonly assumed aggregation structures are brickstone, Figure 7C, and herringbone, Figure 7D.

3D molecular aggregates with translational symmetry are usually known as molecular crystals. Frequently, in these structures is possible to identify lower dimensional subsystems with a preferred interaction between molecular transition dipoles [65]. These directions would determine anisotropy of exciton transport. While on average the exciton transport in a crystal can be incoherent one may expect specific directions with large coherent exciton delocalization.

The above-mentioned structures are only very simple examples of molecular aggregates. For instance, when driven by hydrophobic and hydrophilic forces molecules can aggregate in two-layer tubes of about 10 nanometers in diameter $[17,66]$ or one can use templates like polypeptides or DNA to induce helical structures $[67,68]$.

Because of the huge variety of organic dyes that can aggregate it is possible to create narrow J-band absorption within an arbitrary part of visible and near IR spectrum, see Figure 8.

\subsection{Natural aggregates}

In nature, photosynthetic complexes of plants, phototrophic bacteria and algae are composed of molecular aggregates that efficiently transfer excitons. As an example, in Figure 9 we sketch the energy transfer along the light harvesting system of green sulfur bacteria (GSB). These GSB can be found e.g., in deep sea. By developing a sophisticated light-harvesting antennae structure these organisms adapted to survive at very low light intensities [70, 71]. Energy absorption and transfer in GSB goes through a network of Bchl molecules, Figure 4, aggregated in several
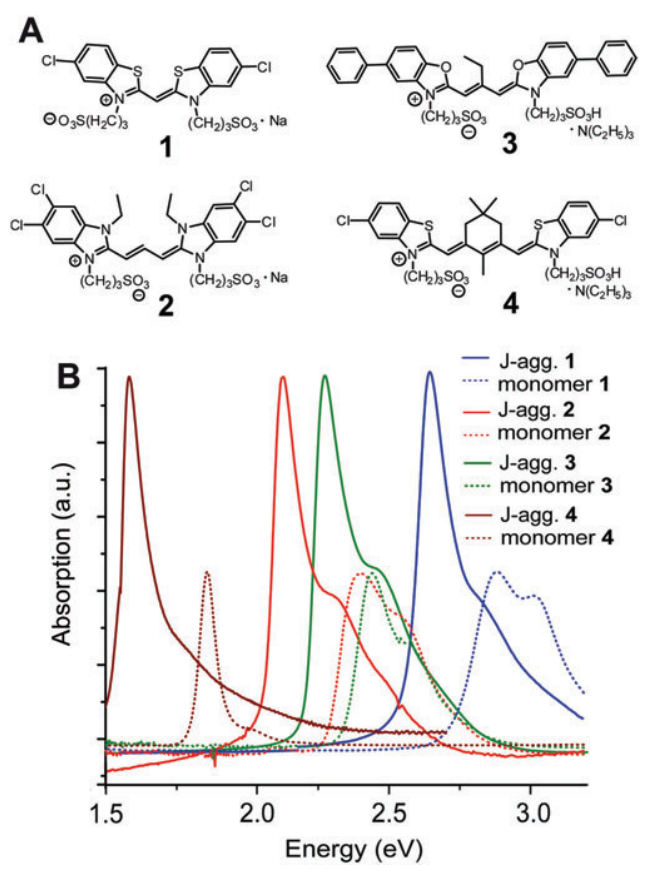

Figure 8 Absorption spectra of four different cyanine dyes in monomeric and aggregated forms illustrating the variability in the aggregate optical properties. Adapted with permission from Ref. [69]. Copyright 2011 American Chemical Society. 


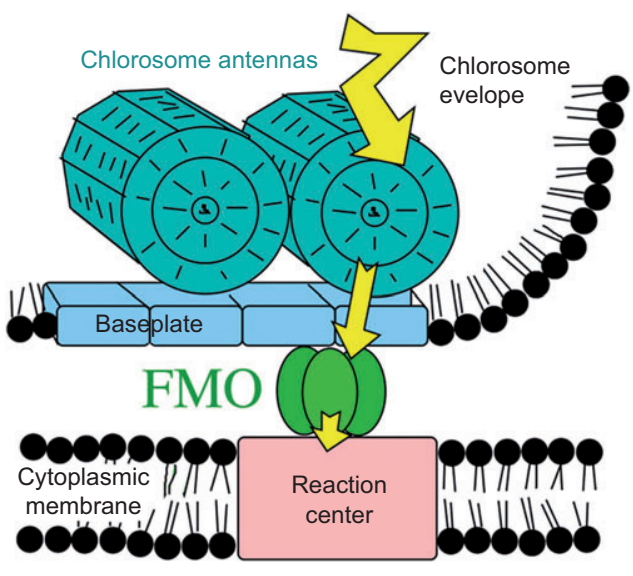

Figure 9 Schematic structure of light-harvesting complex in green sulfur bacteria. The solar light is absorbed by chlorosome antenna aggregates of Bchl molecules. Then, the energy in the form of excitons is transferred through the baseplate and Fenna-MatthewsOlson (FMO) protein complexes to the reaction center, where the charge separation occurs.

types of functional structures - chlorosome antenna, baseplate, Fenna-Matthews-Olson protein complex (FMO), and the reaction center.

The photons are absorbed by the chlorosome - an organelle which is bound to the bacterial membrane and has a size of several hundreds of nanometers. The chlorosome contains a disordered array of cylindrical or ellipsoidal molecular aggregates - antennas. While different types of geometrical and structural disorders complicate the molecular structure characterization of natural aggregates, recent NMR analysis of mutant chlorosome antennas [72] suggested that Bchl molecules in them are arranged in an array of concentric helical structures illustrated in Figure 10A. The Förster coupling between nearest-neighbor Bchl molecules in the chlorosome antenna complexes is of the order of $100 \mathrm{meV}$ and the proposed molecular arrangement of the mutant should result in the formation of a J-band. Both experimental [74] and theoretical studies [75], show that the exciton spreads over a single antennae on the timescale of hundreds of femtoseconds.

Another functional element of GSB light-harvesting structure, is the Fenna-Matthews-Olson (FMO) complex, a protein which is depicted in Figure 10C. FMO plays the role of a molecular wire transferring energy from the chlorosome to the reaction center. It is a trimer containing 8 $\mathrm{Bchl}$ molecules in each subunit. Unlike in the chlorosome antenna, Bchl molecules in FMO are held together by a protein cage, and also the Förster coupling between the molecules is several times weaker. However, from the multiple experimental and theoretical studies it is suggested that the exciton states in FMO are partly delocalized [76-78].

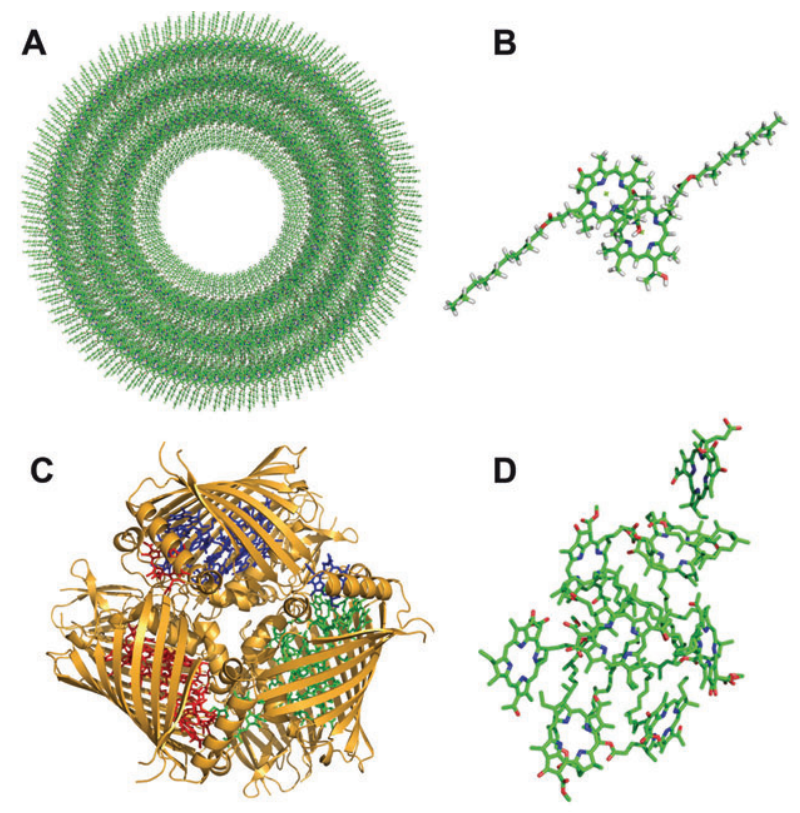

Figure 10 Examples of molecular aggregates in the light-harvesting complex of green sulfur bacteria. (A) Chlorosome antenna complex - a cylindrical or ellipsoidal aggregate of Bchl molecules, several concentric aggregates are enclosed; (B) A Bchl dimer - a unit block of the antenna complex [72]; (C) Fenna-Matthews-Olson (FMO) protein complex - an excitonic "wire"; (D) organization of Bchls in a monomer unit of FMO complex [73].

\section{Experimental characterization of excitonic systems}

Much effort is devoted to obtain the microscopic structure of the various aggregates (which in turn are needed for theoretical models), including the arrangement of the monomers, their spacial and energetic disorder, etc. Beside these conformational aspects there is also large interest in the dynamic properties of excitons such as exciton relaxation and dephasing rates, diffusion coefficients, etc.

X-ray crystallography can be used to obtain lattice properties of molecular aggregates, if the latter either possess an intrinsically long-range order (like molecular crystals or two-dimensional monolayers on substrate [79]) or can be crystallized. For instance, crystallization of photosynthetic light-harvesting complexes [80] had a large impact on the understanding of natural photosynthesis [19]. However, most molecular aggregates cannot be crystallized. In recent years Cryo-Transmission electron microscopy has provided valuable information on the geometrical structure of many aggregates [66, 81-83].

The classical way to obtain information on the geometry of the aggregate is by optical spectroscopy 
(in particular absorption, linear dichroism and circular dichroism) combined with theoretical modeling. For instance, the positions, intensities, polarizations and shapes of the aggregate absorption bands provide information about the strength of intermolecular coupling as well as relative orientations of the monomers [19, 47, 82]. Circular dichroism spectra can be used to analyze chirality of the structures, for instance, in studies of J-aggregate helices and tubes [18, 84-87]. Similarly, the emission spectra can be used to obtain information on the aggregate structure. These optical experiments are often performed under varying environmental conditions like solvent, temperature, concentration of monomers, alignment of the aggregates or pressure [88-90]. More detailed information including separation of homogeneous and inhomogeneous line widths and femtosecond exciton dynamics can be obtained from non-linear 2D spectra [74, 91]. Beside optical spectroscopy of electronic excitations a multitude of various experimental techniques is used to characterize aggregates, e.g., electroabsorption [92], Fourier transform infrared (FTIR) spectroscopy [93], nuclear magnetic resonance NMR [94] and Raman spectroscopy [95].

With optical spectroscopy one probes the structure of energetic levels in the aggregate and phase relations between corresponding electronic transitions, from which one can infer the excitation dynamics in the aggregate. This might be a viable way for small systems, where the exciton is delocalized along the aggregate. However, one would like to follow the dynamics of the exciton also in real space, that means to measure the time-dependent probability to find excitation on a certain monomer. Here in particular, diffusion constants are of interest, which characterize the spreading of the exciton after the initial coherences have died out. Exciton diffusion coefficients cannot be measured directly using existing experimental techniques. The indirect methods include quenching of photoluminescence [96, 97], photocurrent response [98], transient grating [99] and exciton-exciton annihilation [100-103]. Among the state-of-the-art techniques one could mention the recently developed coherent nanoscopy [104], which would allow for spatial resolution comparable with the exciton diffusion length.

\section{Models of exciton dynamics}

While the simple electronic model from Section 2 [see in particular Eq. (5)] already allows us to understand basic properties of molecular aggregates (for instance, the position of the J-band) it is not sufficient to describe important features such as the narrowing of the J-band (in particular in contrast to the broad $\mathrm{H}$-band with vibronic structure). Also, many transport properties, e.g., dependence on temperature [105], cannot be explained. To this end it is necessary to include vibrational modes and the influence of the environment into the description. This can be done in various ways and there exists a multitude of theoretical models in the literature where each is best suited for a particular question and/or situation. For example, the Förster rate theory [24] works well when the timescale of the transfer is very long compared to decoherence and vibrational relaxation of the excitation. In this Section we review the most common models.

\subsection{Static disorder}

Since usually the experiments are performed on ensembles of aggregates where each aggregate experiences a slightly different environment, it is common to average over different "configurations" of the aggregate. Most often the influence of the environment is treated simply as inducing random shifts of the transition frequencies of the monomers. For early works see e.g., [54, 106, 107]. Often, it is assumed that each monomer sees "its own" local environment and the shifts induced by this local environment are uncorrelated from the shifts of the neighboring monomers and disorder of the coupling is ignored. This assumption could be called "uncorrelated diagonal disorder". Sometimes also the positions or couplings of the monomers are treated as random variables (off-diagonal disorder) [107, 108]. For each disorder realization the exciton states are typically no longer delocalized over the full aggregate as in a perfect chain. This localization becomes stronger for larger values of disorder [54, 107, 109]. In the literature, the influence of various forms of correlations has been discussed, see e.g., [54, 110, 111]. To account for temperature a variant of the open system model described below is adapted [112-114], where the environment of the disordered chain can lead to scattering and relaxation between the localized exciton states. Such a model has been successfully used to describe optical and transfer properties of some aggregates [113, 115, 116]. In particular at low temperatures the increase of mobility with increasing temperature could be explained.

In the above formulation the influence of internal vibrations of the monomers is neglected. This seems to be a good approximation for the J-band where one can show that the electronic excitation is to a large extend decoupled from vibrational modes [55, 111, 117]. 


\subsection{Dynamic disorder, Haken-Strobel-Reineker model}

Fluctuations of the monomer transition frequencies or the intermolecular couplings that are fast compared to the exciton transfer time-scale are usually referred to as dynamic disorder. Dynamical fluctuations play a dual role in exciton transport [118-120]. In structures with large static disorder excitons are localized. Dynamic fluctuations in this case remove localization. However, if the fluctuations are strong this results in the dynamical localization of the exciton. A seminal model to treat these fast fluctuations is due to Haken, Strobl and Reineker [121-123], where the dynamical fluctuations are described by real stochastic processes, $\varepsilon_{n}(t)$ and $V_{n m}(t)$ and modeled using the density matrix [121] or stochastic Schrödinger [124] equations. In the original work the stochastic processes have been chosen as white noise, i.e., delta-correlated in time. This model has been shown to capture coherent and incoherent excitation transfer on the same footing. Moreover, one should notice that the equation describing HakenStrobl-Reineker (HRS) model for 1D systems is equivalent to telegrapher's equation, which further reflects the similarity between the exciton propagation in molecular aggregates and a wave propagation in a medium.

The Haken-Strobl-Reineker model, with several extensions, has been utilized to study exciton dynamics in natural and artificial molecular aggregates [120, 123-127]. The drawback of this model is that the exciton population does not relax to thermal equilibrium. There have been many investigations and extensions which have included colored noise and tried to cure the thermalization issue, e.g., $[128,129]$. The original model also does not include the effect of strongly coupled high frequency modes of the monomers which is essential to describe the vibronic structure present in many $\mathrm{H}$-aggregates. The recent development of so-called quantum state diffusion methods $[130,131]$ can be considered as an extension of the HSR model which cures these deficiencies at the cost of colored complex noise.

Figure 11A illustrates how the HRS model can be applied to exciton propagation on a brickstone lattice of TDBC dyes at ambient conditions [124]. The second moment of the exciton wavefunction, Figure 11B shows that the initial propagation on the time scale of 20-30 fs is ballistic or wave-like, which is associated with the quadratic dependence of the second moment on time. For the longer timescale the time dependence is linear, which characterizes a diffusive motion.

\subsection{The vibronic model}

Let us first consider a single monomer, where only the two lowest electronic states are taken into account, see Figure 2. In the Born-Oppenheimer approximation [132] we write the nuclear Hamiltonian of the electronic ground and excited states as

$$
H_{n}^{g / e}=\frac{1}{2} \sum_{j}\left(P_{n j}^{2}+U_{n}^{g / e}\left(Q_{n j}\right)\right)
$$

where $Q_{n j}$ are the (mass-scaled) nuclear coordinates of monomer $n$ and $P_{n j}$ are the corresponding momenta.
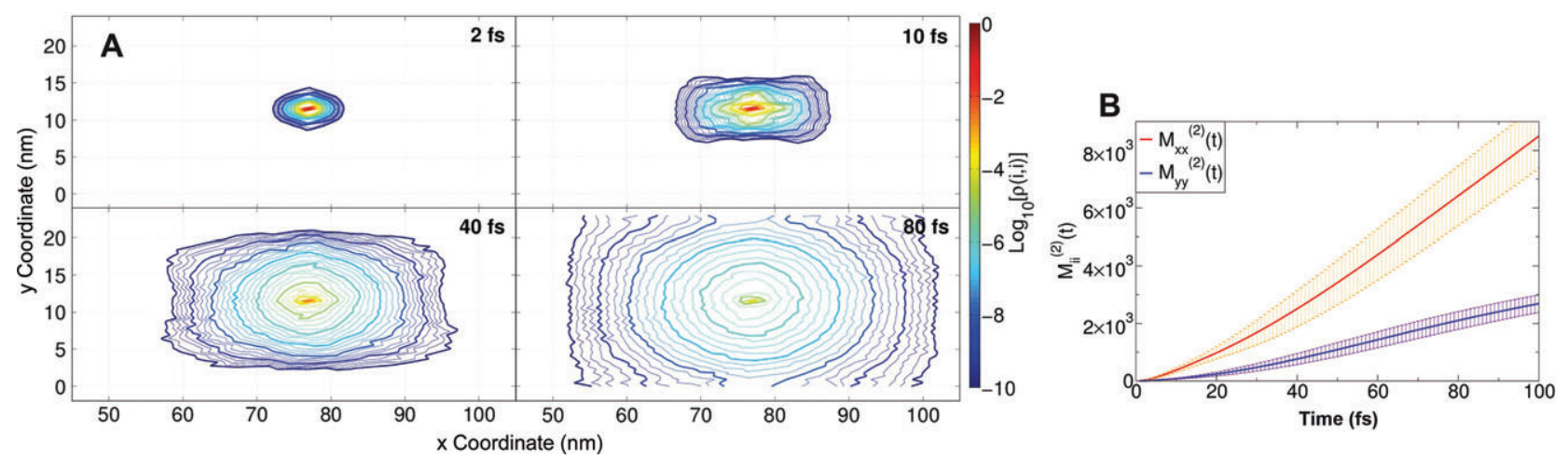

Figure 11 (A) Time dynamics of exciton wave function on a 2D lattice of TDBC molecules subject to static disorder (linewidth $\sigma=70 \mathrm{meV}$ ) and dynamic fluctuations (linewidth $\Gamma=30 \mathrm{meV}$ ) of molecular electronic transitions. At time zero the exciton is localized in the center of the lattice. The contour plots show the population of lattice sites at four different times. (B) Second moments of the wave function corresponding to the exciton dynamics shown in (A). Two different transport regimes can be observed: the ballistic or a wave-like propagation on timescales of 20-30 fs $\left(M^{(2)} \sim t^{2}\right)$ and the diffusive motion $\left(M^{(2)} \sim t\right)$ at longer times. Reprinted with permission from Ref. [124]. Copyright 2012, American Institute of Physics. 
$U^{g / e}\left(Q_{n j}\right)$ is the Born-Oppenheimer potential in the electronic ground/excited state (see Figure 2).

Then, for a molecular aggregate the Hamiltonian in the electronic ground state is given by $H^{g}=\left(\sum_{n=1}^{N} H_{n}^{g}\right)\left|g_{\text {el }}\right\rangle\left\langle g_{\text {el }}\right|$. We denote by $V_{n m}$ the transition dipole-dipole interaction between monomer $n$ and $m$, which, for simplicity, is taken to be independent of nuclear coordinates. The Hamiltonian in the one-exciton manifold, Eq. (4), is then given by

$$
H^{e}=\sum_{n=1}^{N} W_{n}|n\rangle\left\langle n\left|+\sum_{n, m=1}^{N} V_{n m}\right| n\right\rangle\langle m|,
$$

with the "collective" Born-Oppenheimer surfaces $W_{n}=H_{n}^{e}+\sum_{m \neq n}^{N} H_{m}^{g}$. Note that the structure of Eq. (9) is very similar to the purely electronic Hamiltonian (5). The only difference is that now the energies $\varepsilon_{n}$ are replaced by operators for the nuclear motion. Upon transfer of excitation the nuclear wavepacket, according to Figure 2, is no longer in its equilibrium position. Thus the excitation transfer is linked to excitation of nuclear motion. This vibrational model is sometimes combined with the static disorder approach [111, 133, 134]. The vibrational model offers a clear idea regarding why the J-band does not exhibit vibrational structure and the H-band does.

The coupling to vibrations usually slows down the propagation of the exciton, however, it can help to overcome energetic barriers caused by differences in the electronic transition energies. In this way efficient directed transport along a biased chain can be achieved.

Often it is justified to approximate the Born-Oppenheimer potentials, Eq. (8), as harmonic potentials where the surface of the electronically excited state is just shifted relative to that of the ground electronic state, i.e., we have $H_{n}^{g}=\frac{1}{2} \sum_{j=1}^{M}\left(P_{n j}^{2}+\omega_{n j}^{2} Q_{n j}^{2}\right)$ and for the excited electronic state $H_{n}^{e}=\varepsilon_{n}+\frac{1}{2} \sum_{j=1}^{M}\left(P_{n j}^{2}+\omega_{n j}^{2}\left(Q_{n j}-\Delta Q_{n j}\right)^{2}\right)$ where $\varepsilon_{n}$ denotes the energy difference between the minima of the upper and lower potential energy surface and the vibrational frequency of mode $j$ is denoted by $\omega_{n j}$ and the shift between the minima of the excited and ground state harmonic potential is denoted by $\Delta Q_{n j}$. The potential surfaces are sketched in Figure 2 for the case of one mode. This harmonic approximation is widely employed in the literature, e.g., [46, 55, 134-138].

\subsection{Open system approaches}

Open system approaches are closely related to the vibronic model discussed in the previous subsection. In these approaches one typically considers an infinite set of harmonic oscillators forming the bath. It is easy to see that the Hamiltonian (9) can be written as $H^{e}=H_{s y s}+$ $H_{\text {sys-bath }}+H_{\text {bath }}$ with $H_{\text {sys }}$ given by the purely electronic part (5) with transition energies $\varepsilon_{n}$ that already include static overall shifts induced by the environment. The bath is taken as $H_{\text {bath }}=\sum_{n} H_{n}^{g}$ and the system-bath coupling is described by a linear coupling of the electronic excitation of a monomer to the set of vibrational modes, i.e., $H_{\text {sys-bath }}=\sum_{n}\left|\pi_{n}\right\rangle\left\langle\pi_{m}\right| \otimes \sum_{j} \tilde{\kappa}_{n j} Q_{n j}$. The coupling constant $\tilde{\kappa}_{n j}$ describes the coupling of the excitation on monomer $n$ to the harmonic oscillator with frequency $\omega_{n j}$. Typically, the frequency spectrum of bath oscillators is taken to be continuous so that the coupling constant becomes a continuous function of frequency. The open system approaches have been used to describe optical and transfer properties of light harvesting systems $[139,140]$ and can also be applied to explain properties of organic dye aggregates [141]. We note that the HRS model can be considered as a special case of the open system models for which the bath is Markovian (i.e., memoryless).

\subsection{Mixed QM/MM}

With the increase of computer capabilities, in recent years it has become possible to simulate aggregates starting from a microscopic description [142-145]. Since a full quantum mechanical treatment is still out of reach, one uses mixed quantum classical approaches. In these approaches usually the nuclei are propagated classically in the electronic ground state using molecular dynamics. Along these nuclear trajectories one calculates the (time-dependent) transition energies using quantum chemistry methods. These timedependent transition energies can then be used to obtain input parameters for open system models or to use them directly as the energy fluctuations in HSR model. However, both approaches have their problems, since one is restricted to a classical propagation in the electronic ground state.

\subsection{Semi-empirical approach based on polarizabilities}

While the approaches discussed above start from a microscopic description of the exciton-phonon coupling and the environment, in the semi-empirical approaches one can take the standpoint that all the relevant information of the coupling of an electronic transition to other degrees of freedom is already contained in the shape of the absorption spectrum. The absorption spectrum in turn is linked to the polarizabilities of the monomers. 
The induced electric moment of a monomer depends on the local field at that monomer, which is produced by the electric moments of all the other monomers and the external field. This leads to a set of coupled equations from which one can extract, for a given arrangement of the monomers, the polarizability of the aggregate and thus the optical properties. Such equations have been derived using classical treatments [10] or quantum approaches [117, 146]. This method implicitly assumes that the frequency dependent polarizability does not change (beside an overall shift in energy) when going from the monomer to the aggregate. Recently, it has been demonstrated that using measured monomer spectra as input one can accurately describe the band-shape of J- and H-aggregates $[60,147]$. This approach also gives an intuitive explanation of the vibrational structure and the broadenings of the absorption bands of the aggregate.

\subsection{Beyond the single-exciton approximation}

Photonic device applications may require structures operating in a nonlinear response regime, where interactions between excitons cannot be neglected. For example, thin J-aggregate films switches based on optical bistability have been suggested in [30]. At higher intensities, however, an additional loss-channel - exciton-exciton annihilation - appears. The underlying physical process is similar to the Auger effect, followed by a fast non-radiative energy conversion. Within an approximate picture this can be viewed as a process by which two excitons localized on neighboring molecules are combined into a higher-energy electronic excitation of a single molecule. Then, the resulting excitation quickly decays to the lowest excited state or ground state dissipating the exceeding energy through vibrations. A detailed kinematic model for the exciton-exciton annihilation in molecular crystals of different dimensions has been introduced by Suna in [148], and a similar master equation approach for exciton annihilation dynamics in natural photosynthetic complexes has been suggested in [149]. While the microscopic approaches discussed above are still valid, the molecular Hamiltonian (5) should be extended to account for at least two excited states per each monomer $[38,150]$.

\section{Hybrid excitonic structures}

In this section we specifically focus on J-aggregates. Their large absorption and fluorescence cross-sections combined with a narrow line width and a small Stokes shift allow for coherent coupling between excitons and photons in optical cavities or excitons and plasmon modes in metal structures. This property can be utilized as an interface between photonic, plasmonic and excitonic circuits.

\subsection{Exciton-polariton structures}

Most of the studies of strong coupling between excitons in J-aggreagtes and photons have been done using organic microcavities [151-154]. A schematic illustration of an organic microcavity - a planar $\lambda / 2$ cavity with a J-aggregate layer embedded in it - is shown in Figure 12A. Strong coupling of cavity photons with excitons in a J-aggregate result in formation of exciton-polariton modes. This is associated with the splitting of a cavity mode - vacuum Rabi splitting, see Figure 12B.

Within a simple model assuming only one exciton state and a single photon mode the value of the Rabi splitting, $\Omega_{\mathrm{R}}$, is just

$$
\Omega_{\mathrm{R}}=\vec{d}_{\mathrm{X}} \cdot \vec{E}_{\mathrm{v} a c}
$$

where $\vec{d}_{\mathrm{x}}$ is the transition dipole of the narrow exciton transition, $\vec{E}_{\text {vac }}$ is the vacuum field in the position where the J-aggregate is located, and we assume that the vacuum field does not vary substantially on the scale of the aggregate. In J-aggregates the value of the exciton transition dipole scales as with the number $N^{\star}$ of coherently coupled molecules involved $\vec{d}_{\mathrm{x}} \sim \sqrt{N^{\star}}$. The value of $N^{\star}$ is usually much smaller than $N$ - the total number of molecules in the aggregate, since the environment leads to localization of the exciton states. The value of the Rabi splitting observed for J-aggregates strongly coupled with optical cavities ranges from several tens of meV [151, 152] to several hundreds of $\mathrm{meV}[102,153,154]$ depending on
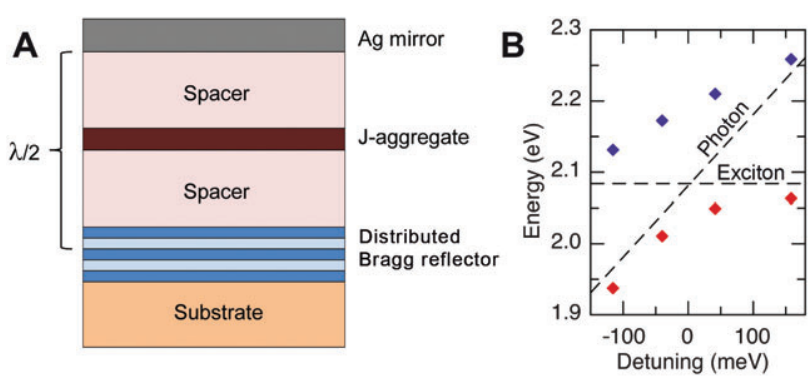

Figure 12 (A) Structure of a planar $\lambda / 2$ organic microcavity with a thin layer of J-aggregates embedded. (B) An example of a cavity photon and a J-aggregate exciton energy level anticrossing. Reprinted with permission from Ref. [102]. Copyright 2010 by the American Physical Society. 
the disorder present in the aggregate and the design of the cavity. Therefore, the splitting can be observed even at room temperatures. The photoluminescence in these structures is usually observed from the lower polariton branch only, due to fast relaxation of polaritons. By detunning the frequency of the cavity mode from the exciton resonance transition one could control the mixture of the exciton and photon and therefore modify its coherence properties making it more photon-like or exciton-like. Electroluminescence from a polariton mode has been demonstrated using a light-emitting-diode structure with a J-aggregate layer [154]. Moreover, lasing from excitonpolariton mode has been shown for cavities with single anthracene crystals [155] and some preliminary results were reported on lasing from cavities with cyanine dye J-aggregates [156]. Large interest has been attracted to the idea of polariton Bose-Einstein condensation (BEC) in organic cavities. This may bring the rich and controversial quantum physics of non-equilibrium polariton condensates [157-160] observed at low temperatures in inorganic systems [161-163] up to room temperatures. However, to the best of our knowledge no confirmed observations of $\mathrm{BEC}$ in organic cavities have been reported yet.

\subsection{Plexciton structures}

A strong coupling of excitons in molecular aggregates with plasmons in noble metals has been demonstrated for both propagating plasmon modes in films [164] as well as localized modes in various types of nanostructures [165, 166] and nanostructure arrays [167]. The hybrid plasmonexciton modes were also named plexcitons [166]. The observed values of the splitting between the plexciton modes are of the order of several hundreds of $\mathrm{meV}$. The linewidth of plasmon modes is usually sufficiently larger than the linewidth of excitons in molecular aggregates (plasmon lifetime is about tens of femtoseconds as compared to picosecond exciton lifetime in J-aggregates). Therefore, the interaction between the exciton and the plasmon modes can frequently be considered as the coupling of a single mode (exciton) to a broader continuum (plasmon). This results in the formation of a Fano resonance $[168,169]$. It is interesting to notice that in the case of a strong optical pumping of plexcitonic structures non-linear Fano effects can be observed [170].

\section{Conclusions}

Aggregates of organic molecules - supramolecular assemblies with strong resonant near-field interactions between electronic transitions - could be exploited in the design of nanophotonic devices at the true nanometer scale. The molecules, forming the aggregates, interact collectively with optical fields, and the absorbed energy is transferred in the form of excitons on a sub-micron scale. The exciton transport within the aggregates possesses coherent properties even at room temperatures, similar to the electromagnetic wave propagation through a medium. Moreover, molecular aggregates can be coupled coherently to photonic and plasmonic structures. While at the present stage there is a sufficient gap between the research communities studying excitonics and photonics, this review calls for merging the knowledge from the two fields.

Acknowledgments: The authors thank Gleb Akselrod and Brian Walker for providing experimental spectra. We also appreciate comments from Alex Govorov on hybrid structures. The Harvard contribution of this work was supported by the Defense Threat Reduction Agency under Contract No HDTRA1-10-1-0046. S. V. acknowledges support from the Center for Excitonics, an Energy Frontier Research Center funded by the U.S. Department of Energy, Office of Science and Office of Basic Energy Sciences under Award Number DE-SC0001088 as well as support from the Defense Advanced Research Projects Agency under award number N66001-10-1-4060.

Received September 1, 2012; accepted December 10, 2012; previously published online February 12, 2013

\section{References}

[1] Yablonovitch E. Inhibited spontaneous emission in solid-state physics and electronics. Phys Rev Lett 1987;58:2059-2062.

[2] Joannopoulos JD, Villeneuve PR, Fan S. Photonic crystals: putting a new twist on light. Nature 1997;386:143-149.

[3] Vahala KJ. Optical microcavities. Nature 2003;424:839-846.

[4] Khitrova G, Gibbs HM, Kira M, Koch SW, Scherer A. Vacuum Rabi splitting in semiconductors. Nat Phys 2006;2:81-90.
[5] Quinten M, Leitner A, Krenn JR, Aussenegg FR. Electromagnetic energy transport via linear chains of silver nanoparticles. Opt Lett 1998;23:1331-1333.

[6] Krenn JR. Nanoparticle waveguides-watching energy transfer. Nat Mater 2003;2:210-211.

[7] Barnes WL, Dereux A, Ebbesen TW. Surface plasmon subwavelength optics. Nature 2003;424:824-830. 
[8] Holtsmark J. Über die Absorption in Na-Dampf. Z Physik A 1925;34:722-729.

[9] Frenkel J. Zur Theorie der Resonanzverbreiterung von Spektrallinien. Z Phys A 1930;59:198-207.

[10] DeVoe H. Optical properties of molecular aggregates. I. Classical model of electronic absorption and refraction. J Chem Phys 1964;41:393-400.

[11] Philpott MR. Some modern aspects of exciton theory. Adv Chem Phys 1973;23:227-341.

[12] Zimanyi EN, Silbey RJ. Unified treatment of coherent and incoherent electronic energy transfer dynamics using classical electrodynamics. J Chem Phys 2010;133:144107, pages 1-10.

[13] Briggs JS, Eisfeld A. Equivalence of quantum and classical coherence in electronic energy transfer. Phys Rev $E$ 2011;83:051911, pages 1-4.

[14] Scheibe G. Über die Veränderlichkeit des Absorptionsspektrums einiger Sensibilisierungsfarbstoffe und deren Ursache. Angew Chem 1936;49:563.

[15] Scheibe G. Über die Veränderlichkeit der Absorptionsspektren in Lösungen und die Nebenvalenzen als ihre Ursache. Angew Chem 1937;50:212-219.

[16] Jelley EE. Spectral absorption and fluorescence of dyes in the molecular state. Nature 1936;138:1009-1010.

[17] Kirstein S, Daehne S. J-aggregates of amphiphilic cyanine dyes: self-organization of artificial light harvesting complexes. Int J Photoenergy 2006;20363, pages 1-21.

[18] Norden B. Linear and circular-dichroism of polymeric pseudoisocyanine. J Phys Chem 1977;81:151-159.

[19] van Amerongen H, Valkunas L, van Grondelle R. Photosynthetic excitons. World Scientific, Singapore 2000

[20] Scheibe G. Über den Mechanismus der Sensibilisierung photochemischer Reaktionen durch Farbstoffe, insbesondere der Assimilation. Naturwissenschaften 1937;49:795

[21] Franck J, Teller E. Migration and photochemical action of excitation energy in crystals. J Chem Phys 1938;6:861-872.

[22] Frenkel J. On the transformation of light into heat in solids. I. Phys Rev 1931;37:17-44.

[23] Förster T. Zwischenmolekulare Energiewanderung und Fluoreszenz. Ann Phys (Leipzig) 1948;437:55-75.

[24] Förster T. Delocalized excitation and excitation transfer; in Sinanoğlu, editor, Modern Quantum Chemistry; chapter III B 1, pages 93-137; Academic Press 1965.

[25] Tani T. J-aggregates in spectral sensitization of photographic materials; in T. Kobayashi, editor, J-Aggregates. World Scientific 1996

[26] Takechi K, Sudeep PK, Kamat PV. Harvesting infrared photons with tricarbocyanine dye clusters. J Phys Chem B 2006;110:16169-16173.

[27] Reers M, Smith TW, Chen LB. J-aggregate formation of a carbocyanine as a quantitative fluorescent indicator of membrane-potential. Biochemistry 1991;30:4480-4486

[28] Horn D, Rieger J. Organic nanoparticles in the aqueous phase-theory, experiment, and use. Angew Chem Int Ed 2001;40:4331-4361.

[29] Higgins DA, Kerimo J, Vanden Bout DA, Barbara PF. A molecular yarn: near-field optical studies of self-assembled, flexible, fluorescent fibers. J Am Chem Soc 1996;118:4049-4058.

[30] Malyshev VA, Glaeske H, Feller KH. Intrinsic optical bistablility of an ultrathin film consisting of oriented linear aggregates. J Chem Phys 2000;113:1170-1176.
[31] Möbius D, Kuhn H. Energy transfer in monolayers with cyanine dye Scheibe aggregates, J Appl Phys 1988;64:5138-5141.

[32] Akselrod GM, Walker BJ, Tisdale WA, Bawedi MG, Bulović V. Twenty-fold enhancement of molecular fluoresence by coupling to a J-aggredate critically coupled resonator. ACS Nano 2012;6:467-471.

[33] Dähne S. Nanostrukturierte J-Aggregate als potenzielle Lichtsammelsysteme für Photosynthesen. Bunsen-Magazin 2002;4:81-92.

[34] Würthner F, Kaise TE, Saha-Möller CR. J-aggregates: from serendipitous discovery to supramolecular engineering of functional dye materials. Angew Chem Int Ed 2011;50: 3376-3410.

[35] Davydov A. Theory of molecular excitons. McGraw-Hill 1962.

[36] Kobayashi T, editor. J-aggregates. World Scientific 1996.

[37] Scholes GD, Rumbles G. Excitons in nanoscale systems. Nat Mater 2006;5:683-696.

[38] Kühn O, Lochbrunner S. Quantum efficiency in complex systems, part II: from molecular aggregates to organic solar cells; Semiconductors and Semimetals 85; chapter Quantum Dynamics and Spectroscopy of Excitons in Molecular Aggregates, pages 47-81; Academic Press, San Diego (2011).

[39] Medvedev ES, Osherov VI. Radiationless transitions in polyatomic molecules. volume 57; Springer-Verlag 1995.

[40] Bradley M, Tischler J, Bulović V. Layer-by-layer J-aggregate thin films with a peak absorption constant of 106 wavenumbers. Adv Mater 2005;17:1881-1886.

[41] Czikklely V, Forsterling HD, Kuhn H. Extended dipole model for aggregates of dye molecules. Chem Phys Lett 1970;6:207-210.

[42] Krueger BP, Scholes GD, Fleming GR. Calculation of couplings and energy-transfer pathways between the pigments of LH2 by the ab-initio transition density cube method. J Phys Chem B 1998;102:5378-5386.

[43] Kopainsky B, Hallermeier JK, Kaiser W. The first step of aggregation of PIC: the dimerization. Chem Phys Lett 1981;83:498-502.

[44] Fulton RL, Gouterman M. Vibronic coupling. II. Spectra of dimers. J Chem Phys 1964;41:2280-2286.

[45] Kühn 0, Renger T, May V. Theory of exciton-vibrational dynamics in molecular dimers. Chem Phys 1996;204:99-114.

[46] Seibt J, Lohr A, Würthner F, Engel V. Circular dichroism and absorption spectroscopy of merocyanine dimer aggregates: molecular properties and exciton transfer dynamics from time-dependent quantum calculations. Phys Chem Phys 2007;9:6214-6218.

[47] Eisfeld A. A simple method to obtain information on the conformation of dipole-dipole coupled dimers. Chem Phys Lett 2007;445:321-324.

[48] Womick JM, Moran AM. Exciton coherence and energy transport in the light-harvesting dimers of allophycocyanin. J Phys Chem B 2009;113:15747-15759.

[49] Guthmuller J, Zutterman F, Champagne B. Multimode simulation of dimer absorption spectra from first principles calculations: application to the 3,4,9,10-perylenetetracarboxylic diimide dimer. J Chem Phys 2009;131:154302, pages 1-8.

[50] Moffitt W, Fitts DD, Kirkwood JG. Critique of the theory of optical activity of helical polymers. Proc Natl Acad Sci USA 1957;43:723-730.

[51] Holstein T. Studies of polaron motion: part II. The "small" polaron. Ann Phys (NY) 1959;8:343-389. 
[52] Hofelich F. Die Bewegung eines Exzitons entlang eines Polymers unter dem Einflu $\beta$ der Gitterschwingungen. Z Phys B 1966;5:208.

[53] Bierman A. Exciton wave packet localization on an impurity. J Chem Phys 1970;52:4987-4995.

[54] Knapp EW. Lineshapes of molecular aggregates, exchange narrowing and intersite correlation. Chem Phys 1984;85:73-82.

[55] Scherer POJ, Fischer SF. On the theory of vibronic structure of linear aggregates. Application to pseudoisocyanin (PIC). Chem Phys 1984;86:269.

[56] Spano FC. Fermion excited states in one-dimensional molecular aggregates with site disorder: nonlinear optical response. Phys Rev Lett 1991;67:3424-3427.

[57] Knoester J. Nonlinear optical line shapes of disordered molecular aggregates: motional narrowing and the effect of intersite correlations. J Chem Phys 1993;99:8466-8479.

[58] Hoffmann M, Schmidt K, Fritz T, Hasche T, Agranovich VM, Leo K. The lowest energy Frenkel and charge-transfer excitons in quasi-one-dimensional structures: application to MePTCDI and PTCDA crystals. Chem Phys 2000;258:73-96.

[59] May V, Kühn O. Charge and energy transfer dynamics in molecular systems. WILEY-VCH 2000.

[60] Eisfeld A, Briggs JS. The J- and H-bands of organic dye aggregates. Chem Phys 2006;324:376-384.

[61] Pavinatto F, Gameiro A Jr., Hidalgo A, Dinelli L, Romualdo L, Batista A, Neto NB, Ferreira M, Oliveira 0 Jr. Langmuir and Langmuir-Blodgett (LB) films of tetrapyridyl metalloporphyrins. Appl Surf Sci 2008;254:5946-5952.

[62] Proehl H, Nitsche R, Dienel T, Leo K, Fritz T. In situ differential reflectance spectroscopy of thin crystalline films of PTCDA on different substrates. Phys Rev B 2005;71:165207, pages 1-14.

[63] Misawa K, Ono H, Minoshima K, Kobayashi T. New fabrication method for highly oriented J aggregates dispersed in polymer films. Appl Phys Lett 1993;63:577-579.

[64] Mishra A, Behera RK, Behera PK, Mishra BK, Behera GB. Cyanines during the 1990s: a review. Chem Rev 2000;100:1973-2011.

[65] Schwoerer M, Wolf H. Organic molecular solids. Wiley-VCH 2006.

[66] Eisele DM, Cone CW, Bloemsma EA, Vlaming SM, van der Kwaak CGF, Silbey RJ, Bawendi MG, Knoester J, Rabe JP, Vanden Bout DA. Utilizing redox-chemistry to elucidate the nature of exciton transitions in supramolecular dye nanotubes. Nat Chem 2012;4:655-662.

[67] Stryer L, Blout ER. Optical rotatory dispersion of dyes bound to macromoleculescationic dyespolyglutamic acid complexes. J Am Chem Soc 1961;83:1411-1418.

[68] Seifert JL, Connor RE, Kushon SA, Wang M, Armitage BA. Spontaneous assembly of helical cyanine dye aggregates on DNA nanotemplates. J Am Chem Soc 1999;121:2987-2995.

[69] Walker BJ, Dorn A, Buloví V, Bawendi MG. Color-selective photocurrent enhancement in coupled J-aggregate/nanowires formed in solution. Nano Lett 2011;11:2655-2659.

[70] Overmann J, Cypionka H, Pfennig N. An extremely low-lightadapted phototrophic sulfur bacterium from the Black Sea. Limnol Oceanogr 1992;37:150-155.

[71] Beatty JT, Overmann J, Lince MT, Manske AK, Lang AS, Blankenship RE, Dover CLV, Martinson TA, Plumley FG. An obligately photosynthetic bacterial anaerobe from a deep-sea hydrothermal vent. Proc Natl Acad Sci USA 2005;102:9306-9310.
[72] Ganapathy S, Oostergetel GT, Wawrzyniak PK, Reus M, Chew AGM, Buda F, Boekema EJ, Bryant DA, Holzwarth AR, de Groot HJM. Alternating syn-anti bacteriochlorophylls form concentric helical nanotubes in chlorosomes. Proc Natl Acad Sci USA 2009;106:8525-8530.

[73] Tronrud DE, Wen J, Gay L, Blankenship RE. The structural basis for the difference in absorbance spectra for the FMO antenna protein from various green sulfur bacteria. Photosynth Res 2009;100:79-87.

[74] Dostal J, Mancal T, Augulis R, Vacha F, Psencik J, Zigmantas D. Two-dimensional electronic spectroscopy reveals ultrafast energy diffusion in chlorosomes. J Am Chem Soc 2012;134:11611-11617.

[75] Fujita T, Brookes JC, Saikin SK, Aspuru-Guzik A. Memoryassisted exciton diffusion in the chlorosome light-harvesting antenna of green sulfur bacteria. J Phys Chem Lett 2012;3:2357-2361.

[76] Milder M, Brüggemann B, van Grondelle R, Herek J. Revisiting the optical properties of the FMO protein. Photosynth Res 2010;104:257-274.

[77] Renger T, May V. Ultrafast exciton motion in photosynthetic antenna systems: the FMO-Complex. J Phys Chem A 1998;102:4381-4391.

[78] Adolphs J, Renger T. How proteins trigger excitation energy transfer in the FMO complex of green sulfur bacteria. Biophys J 2006;91:2778-2797.

[79] Müller M, Ikonomov J, Sokolowski M. Structure of epitaxial layers of $\mathrm{KCl}$ on $\mathrm{Ag}(100)$. Surf Sci 2011;605:1090-1094.

[80] Olson JM. The FMO protein. Photosynth Res 2004;80:181-187.

[81] von Berlepsch H, Böttcher C, Ouart A, Burger C, Dähne S, Kirstein S. Supramolecular structures of J-aggregates of carbocyanine dyes in solution. J Phys Chem B 2000;104:5255-5262.

[82] Vlaming SM, Augulis R, Stuart MCA, Knoester J, van Loosdrecht PHM. Exciton spectra and the microscopic structure of self-assembled porphyrin nanotubes. J Phys Chem B 2009;113:2273-2283.

[83] Oostergetel GT, Reus M, Chew AGM, Bryant DA, Boekema EJ, Holzwarth AR. FEBS Lett 2007;581:5435-5439.

[84] Spitz C, Dähne S, Ouart A, Abraham H-W. Proof of chirality of J-aggregates spontaneously and enantioselectively generated from achiral dyes. J Phys Chem B 2000;104:8664-8669.

[85] Didraga C, Klugkist JA, Knoester J. Optical properties of helical cylindrical molecular aggregates: the homogeneous limit. J Phys Chem B 2002;106:11474-11486.

[86] Didraga C, Pugžlys A, Hania PR, von Berlepsch H, Duppen K, Knoester J. Structure, spectroscopy, and microscopic model of tubular carbocyanine dye aggregates. J Phys Chem B 2004;108:14976-14985.

[87] Eisfeld A, Kniprath R, Briggs J. Theory of the absorption and circular dichroism spectra of helical molecular aggregates. J Chem Phys 2007;126:104904, pages 1-13.

[88] Renge I, Wild UP. Solvent, temperature, and excitonic effects in the optical spectra of pseudoisocyanine monomer and J-aggregates. J Phys Chem A 1997;101:7977-7988.

[89] Lindrum M, Chan IY. High pressure investigation of absorption spectra of J-aggregates. J Chem Phys 1996;104:5359-5364.

[90] Kangur L, Leiger K, Freiberg A. Evidence for high-pressureinduced rupture of hydrogen bonds in LH2 photosynthetic antenna pigment-protein complexes. J Phys Conf Ser 2008;121:112004, pages 1-5. 
[91] Stiopkin I, Brixner T, Yang M, Fleming GR. Heterogeneous exciton dynamics revealed by two-dimensional optical spectroscopy. J Phys Chem B 2006;110:20032-20037.

[92] Luchowski R, Krawczyk S. Stark effect spectroscopy of exciton states in the dimer of acridine orange. Chem Phys 2003;293:155-166.

[93] Ilharco LM, de Barros RB. Aggregation of pseudoisocyanine iodide in cellulose acetate films: structural characterization by FTIR. Langmuir 2000;16:9331-9337.

[94] van Rossum BJ, Steensgaard DB, Mulder FM, Boender GJ, Schaffner K, Holzwarth AR, de Groot HJM. A refined model of the chlorosomal antennae of the green bacterium Chlorobium tepidum from proton chemical shift constraints obtained with high-field 2-D and 3-D MAS NMR dipolar correlation spectroscopy. Biochem 2001;40: 1587-1595.

[95] Leng WN, Würthner F, Kelley AM. Resonance Raman intensity analysis of merocyanine dimers in solution. J Phys Chem B 2004;108:10284-10294.

[96] Lyons B, Monkman A. The role of exciton diffusion in energy transfer between polyfluorene and tetraphenyl porphyrin. Phys Rev B 2005;71:235201, pages 1-5.

[97] Lunt RR, Giebink NC, Belak AA, Benziger JB, Forrest SR. Exciton diffusion lengths of organic semiconductor thin films measured by spectrally resolved photoluminescence quenching. J Appl Phys 2009;105:053711, pages 1-7.

[98] Ghosh AK, Feng T. Merocynanine organic solar cells. J Appl Phys 1978;49:5982-5989.

[99] Salcedo J, Siegman A, Dlott D, Fayer M. Dynamics of energy transport in molecular crystals: the picosecond transientgrating method. Phys Rev Lett 1978;41:131-134.

[100] Moll J, Harrison WJ, Brumbaugh DV, Muenter AA. Exciton annihilation in J-aggregates probed by femtosecond fluorescence upconversion. J Phys Chem A 2000;104: 8847-8854.

[101] Shaw PE, Ruseckas A, Peet J, Bazan GC, Samuel IDW. Exciton-exciton annihilation in mixed-phase polyfluorene films. Adv Funct Mater 2010;20:155-161.

[102] Akselrod G, Tischler Y, Young E, Nocera D, Bulovic V. Exciton-exciton annihilation in organic polariton microcavities. Phys Rev B 2010;82:113106, pages 1-4.

[103] Marciniak H, Li X-Q, Würthner F, Lochbrunner S. One-dimensional exciton diffusion in perylene bisimide aggregates. J Phys Chem A 2011;115:648-654.

[104] Aeschlimann M, Brixner T, Fischer A, Kramer C, Melchior P, Pfeiffer W, Schneider C, Strüber C, Tuchscherer P, Voronine DV. Coherent two-dimensional nanoscopy. Science 2011;333:1723-1726.

[105] Scheblykin IG. Temperature dependence of exciton transport in J-aggredates; in J-aggregates. World Scientific 2012.

[106] Schreiber M, Toyozawa Y. Numerical experiments on the absorption lineshape of the exciton under latticevibrations. I. The overall lineshape. J Phys Soc Jap 1982;51:1528-1536.

[107] Fidder H, Knoester J, Wiersma DA. Optical properties of disordered molecular aggregates: a numerical study. J Chem Phys 1991;95:7880-7890.

[108] Malyshev VA, Dominguez-Adame F. Motional narrowing effect in one-dimensional Frenkel chains with configurational disorder. Chem Phys Lett 1999;313:255-260.
[109] Malyshev VA. Localization length of a 1-D exciton and temperature dependence of the radiative lifetime in frozen dye solutions with J-aggregates. Opt Spectrosc 1991;71:505-506.

[110] Domínguez-Adame F, Malyshev VA. Frenkel excitons in one-dimensional systems with correlated disorder. J Lumin 1999;83-4:61-67.

[111] Walczak P, Eisfeld A, Briggs JS. Exchange narrowing of the J-band of molecular dye aggregates. J Chem Phys 2008;128:044505, pages 1-12.

[112] Heijs DJ, Malyshev VA, Knoester J. Decoherence of excitons in multichromophore systems: thermal line broadening and destruction of superradiant emission. Phys Rev Lett 2005;95:177402, pages 1-4.

[113] Bednarz M, Malyshev VA, Knoester J. Temperature dependent fluorescence in disordered frenkel chains: interplay of equilibration and local band-edge level structure. Phys Rev Lett 2003;91:217401, pages 1-4.

[114] Vlaming SM, Malyshev VA, Knoester J. Nonmonotonic energy harvesting efficiency in biased exciton chains. J Chem Phys 2007;127:154719, pages 1-8.

[115] Tomioka A, Miyano K. Numerical study of excitons in a two-dimensional organic dye aggregate. Phys Rev B 1996;54:2963-2967.

[116] Gallos LK, Pimenov AV, Scheblykin IG, Van der Auweraer M, Hungerford G, Varnavsky OP, Vitukhnovsky AG, Argyrakis P. A kinetic model for J-aggregate dynamics. J Phys Chem B 2000;104:3918-3923.

[117] Briggs JS, Herzenberg A. Bandshapes in polymer spectra. Mol Phys 1971;21:865-879.

[118] Logan DE, Wolynes PG. Localizability and dephasing of dipolar excitons in topologically disordered systems. J Chem Phys 1987;87:7199-7207.

[119] Evensky DA, Scalettar RT, Wolynes PG. Localization and dephasing effects in a time-dependent Anderson Hamiltonian. J Phys Chem 1990;94:1149-1154.

[120] Rebentrost P, Mohseni M, Kassal I, Lloyd S, Aspuru-Guzik A. Environment-assisted quantum transport. New J Phys 2009;11:033003, pages 1-12.

[121] Haken H, Reineker P. The coupled coherent and incoherent motion of excitons and its influence on the line shape of optical absorption. Z Phys 1972;249:253-268.

[122] Haken H, Strobl G. An exactly solvable model for coherent and incoherent exciton motion. Z Phys 1973;262:135-148.

[123] Reineker P, Krenke VM. Exciton dynamics in molecular crystals and aggregates. Volume 94 of Springer tracts in modern physics. Springer Verlag 1982.

[124] Valleau S, Saikin SK, Yung M-H, Aspuru-Guzik A. Exciton transport in thin-film cyanine dye J-aggregates. J Chem Phys 2012;137:034109, pages 1-13.

[125] Madhukar A, Post W. Exact solution for the diffusion of a particle in a medium with site diagonal and off-diagonal dynamic disorder. Phys Rev Lett 1977;39:1424-1427.

[126] Plenio MB, Huelga SF. Dephasing-assisted transport: quantum networks and biomolecules. New J Phys 2008;10:113019, pages 1-14.

[127] Eisfeld A, Briggs JS. Classical master equation for excitonic transport under the influence of an environment. Phys Rev $E$ 2012;85:046118.

[128] Čápek V. Generalized Haken-Strobl-Reineker model of excitation transfer. Z Phys B 1985;60:101-105. 
[129] Rahman TS, Knox RS, Kenkre VM. Theory of depolarization of fluorescence in molecular pairs. Chem Phys 1979;44:197-211.

[130] Diósi L, Strunz WT. The non-Markovian stochastic Schrödinger equation for open systems. Phys Lett A 1997;235:569-573.

[131] Roden J, Eisfeld A, Wolff W, Strunz WT. Influence of complex exciton-phonon coupling on optical absorption and energy transfer of quantum aggregates. Phys Rev Lett 2009;103:058301, pages 1-4.

[132] Azumi T, Matsuzaki K. What does the term "Vibronic Coupling” Mean? Photochem Photobiol 1977;25:315-326.

[133] Spano FC. Temperature dependent emission in disordered herringbone aggregates of conjugated oligomers. Phys Rev B 2005;71:235208, pages 1-13.

[134] van Dijk L, Spano FC, Bobbert PA. Theory of exciton dynamics in molecular aggregates in presence of polaronic effects. Chem Phys Lett 2012;529:69-73.

[135] Merrifield RE. Theory of the vibrational structure of molecular excition states. J Chem Phys 1964;40:445-450.

[136] Perrin MH, Gouterman M. Vibronic coupling. IV. Trimers and trigonal molecules. J Chem Phys 1967;46:1019-1028.

[137] Eisfeld A, Braun L, Strunz WT, Briggs JS, Beck J, Engel V. Vibronic energies and spectra of molecular dimers. J Chem Phys 2005;122:134103, pages 1-10.

[138] Seibt J, Winkler T, Renziehausen K, Dehm V, Würthner F, Meyer $H-D$, Engel V. Vibronic transitions and quantum dynamics in molecular oligomers: a theoretical analysis with an application to aggregates of perylene bisimides. J Phys Chem A 2009;113:13475-13482.

[139] Ishizaki A, Fleming GR. Unified treatment of quantum coherent and incoherent hopping dynamics in electronic energy transfer: reduced hierarchy equation approach. J Chem Phys 2009;130:234111, pages 1-10.

[140] Moix J, Wu J, Huo P, Coker D, Cao J. Efficient energy transfer in light-harvesting systems, III: The influence of the eighth bacteriochlorophyll on the dynamics and efficiency in FMO. J Phys Chem Lett 2011;2:3045-3052.

[141] Roden J, Strunz WT, Eisfeld A. Spectral properties of molecular oligomers. A non-Markovian quantum state diffusion approach. Int J Mod Phys B 2010;24:5060-5067.

[142] Schulten K, Tesch M. Coupling of protein motion to electron transfer: molecular dynamics and stochastic quantum mechanics study of photosynthetic reaction centers. Chem Phys 1991;158:421-446.

[143] Damjanović A, Kosztin I, Kleinekathöfer U, Schulten K. Excitons in a photosynthetic light-harvesting system: $a$ combined molecular dynamics, quantum chemistry, and polaron model study. Phys Rev E 2002;65:031919, pages 1-24.

[144] Olbrich C, Jansen TLC, Liebers J, Aghtar M, Strümpfer J, Schulten K, Knoester J, Kleinekathöfer U. From atomistic modeling to excitation transfer and two-dimensional spectra of the FMO light-harvesting complex. J Phys Chem B 2011;115:8609-8621.

[145] Shim S, Rebentrost P, Valleau S, Aspuru-Guzik A. Atomistic study of the long-lived quantum coherences in the Fenna-Matthews-Olson complex. Biophys J 2012;102: 649-660.

[146] McLachlan AD, Ball MA. Hypochromism and optical rotation in helical polymers. Mol Phys 1964;8:581-595.

[147] Eisfeld A, Briggs JS. The shape of the J-band of pseudoisocyanine. Chem Phys Lett 2007;446:354-358.
[148] Suna A. Kinematics of exciton-exciton annihilation in molecular crystals. Phys Rev B 1970;1:1716-1739.

[149] Paillotin G, Swenberg CE, Breton J, Geacintov NE. Analysis of picosecond laser-induced fluorescence phenomena in photosynthetic membranes utilizing a master equation approach. Biophys J 1979;25:513-533.

[150] Knoester J, Spano FC. Unusual behavior of two-photon absorption from three-level molecules in a one-dimensional lattice. Phys Rev Lett 1995;74:2780-2783.

[151] Lidzey DG, Bradley DDC, Virgili T, Armitage A, Skolnick MS, Walker S. Room temperature polariton emission from strongly coupled organic semiconductor microcavities. Phys Rev Lett 1999;82:3316-3319.

[152] Schouwink P, Berlepsch HV, Dähne L, Mahrt RF. Observation of strong exciton-photon coupling in an organic microcavity. Chem Phys Lett 2001;344:352-356.

[153] Hobson PA, Barnes WL, Lidzey DG, Gehring GA, Whittaker DM, Skolnick MS, Walker S. Strong exciton-photon coupling in a low-Q all-metal mirror microcavity. Appl Phys Lett 2002;81:3519-3521.

[154] Tischler JR, Bradley MS, V. Bulović, Song JH, Nurmikko A. Strong coupling in a microcavity LED. Phys Rev Lett 2005;95:036401, pages 1-4.

[155] Kena-Cohen S, Forrest SR. Room-temperature polariton lasing in an organic single-crystal microcavity. Nat Photon 2010;4:371-375.

[156] Akselrod GM, Young ER, Bradley MS, Bulovic V. Room temperature organic polariton lasing by intra-cavity pumping; in TSRC workshop on spontaneous coherence and collective dynamics, July 4-8, 2011-Telluride, Colorado 2011.

[157] Moskalenko SA, Snoke DW. Bose-Einstein condensation of excitons and biexcitons and coherent nonlinear optics with excitons. Cambridge University Press 2000.

[158] Deng H, Haug H, Yamamoto Y. Exciton-polariton Bose-Einstein condensation. Rev Mod Phys 2010;82:1489-1537.

[159] Butov LV, Kavokin AV. The behaviour of exciton-polaritons. Nat Photon 2012;6:2.

[160] Deveaud-Plédran B. The behaviour of exciton-polaritons. Nat Photon 2012;6:205

[161] Kasprzak J, Richard M, Kundermann S, Baas A, Jeambrun JM], Keeling P, Marchetti FM, Szymańska MH, André R, Staehli JL, Savona V, Littlewood PB, Deveaud B, Dang LS. Bose-Einstein condensation of exciton polaritons. Nature 2006;443:409-414.

[162] Utsunomiya S, Tian L, Roumpos G, Lai CW, Kumada N, Fujisawa T, Kuwata-Gonokami M, Loffler A, Hofling S, Forchel A, Yamamoto Y. Observation of Bogoliubov excitations in exciton-polariton condensates. Nat Phys 2008;4:700-705.

[163] Amo A, Sanvitto D, Laussy FP, Ballarini D, Valle ED, Martin MD, Lemaitre A, Bloch J, Krizhanovskii DN, Skolnick MS, Tejedor C, Vina L. Collective fluid dynamics of a polariton condensate in a semiconductor microcavity. Nature 2009;457:291-295.

[164] Bellessa J, Bonnand C, Plenet JC, Mugnier J. Strong coupling between surface plasmons and excitons in an organic semiconductor. Phys Rev Lett 2004;93:036404, pages 1-4.

[165] Sugawara Y, Kelf TA, Baumberg JJ, Abdelsalam ME, Bartlett PN. Strong coupling between localized plasmons and organic excitons in metal nanovoids. Phys Rev Lett 2006;97:266808, pages 1-4.

[166] Fofang NT, Park T-H, Neumann O, Mirin NA, Nordlander P, Halas NJ. Plexcitonic nanoparticles: plasmon-exciton coupling in nanoshell-J-aggregate complexes. Nano Lett 2008;8:3481. 
[167] Bellessa J, Symonds C, Vynck K, Lemaitre A, Brioude A, Beaur L, Plenet JC, Viste P, Felbacq D, Cambril E, Valvin P. Giant Rabi splitting between localized mixed plasmon-exciton states in a two-dimensional array of nanosize metallic disks in an organic semiconductor. Phys Rev B 2009;80:033303, pages 1-4.

[168] Fano U. Effects of configuration interaction on intensities and phase shifts. Phys Rev 1961;118:1866-1878.
[169] Zhang W, Govorov AO, Bryant GW. Semiconductor-metal nanoparticle molecules: hybrid excitons and non-linear Fano effect. Phys Rev Lett 1997;97:146804, pages 1-4.

[170] Fofang NT, Grady NK, Fan Z, Govorov AO, Halas NJ. Plexciton dynamics: exciton-plasmon coupling in a J-aggregate-Au nanoshell complex provides a mechanism for nonlinearity. Nano Lett 2011;11:1556-1560. 